Supporting Information for

\title{
Tuning Oxygen content of reduced graphene oxide and effects on its properties
}

Wei Liu ${ }^{1}$, Giorgio Speranza ${ }^{1,2,3 *}$

${ }^{1}$ Fondazione Bruno Kessler, via Sommarive 18, 38123 Trento, Italy

${ }^{2}$ Department of Industrial Engineering, University of Trento, via Sommarive 9, 38123 Trento, Italy

${ }^{3}$ Istituto di Fotonica e Nanotecnologie, IFN-CNR, via alla Cascata 56/C, 38123 Trento, Italy

\footnotetext{
*Corresponding author. Email address:speranza@fbk.eu
} 
XPS analysis

(A)

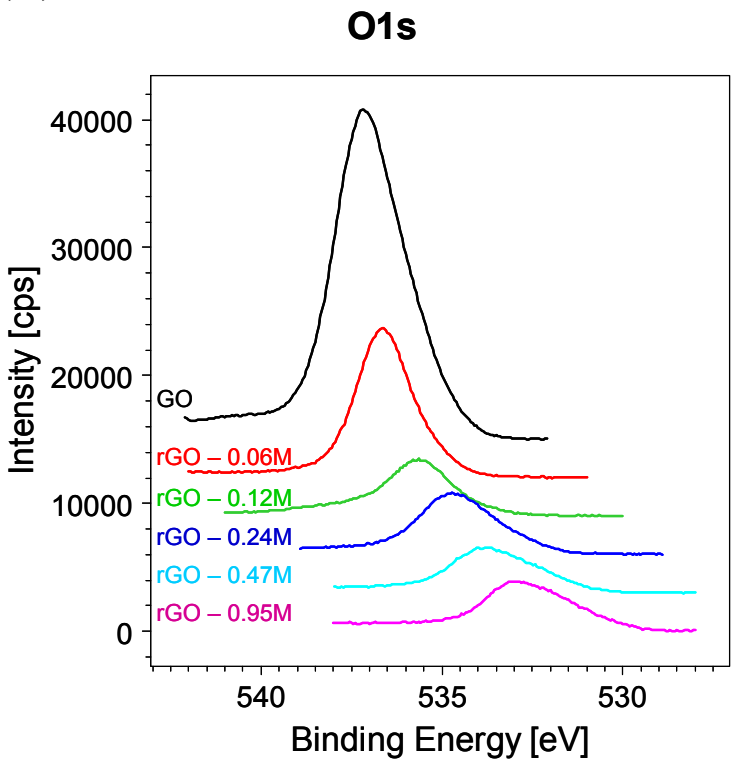

(C)

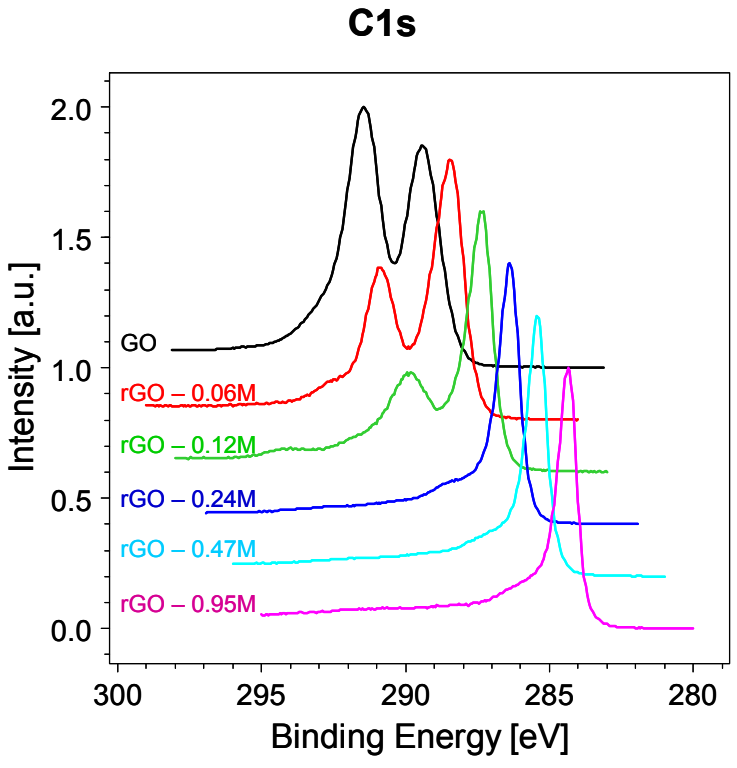

(B)

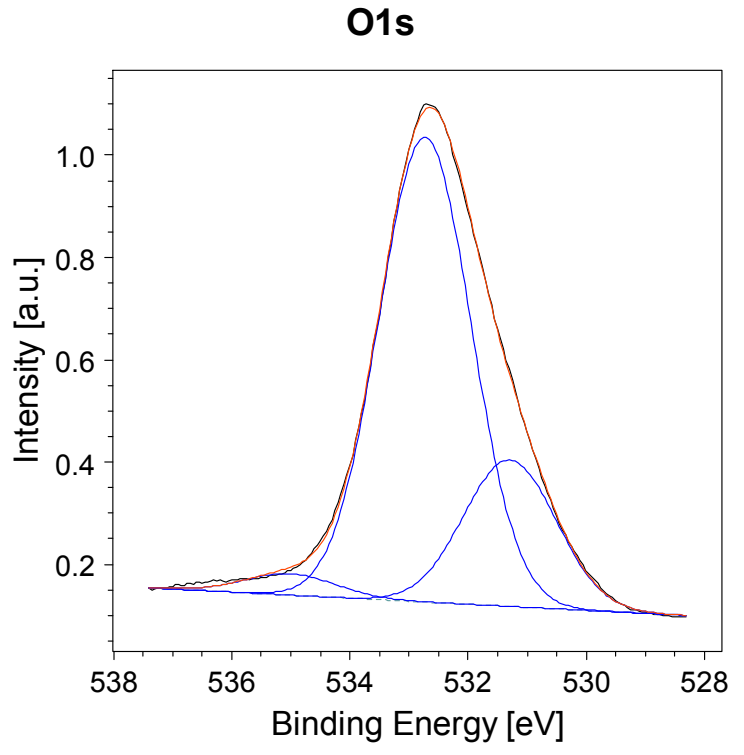

(D)

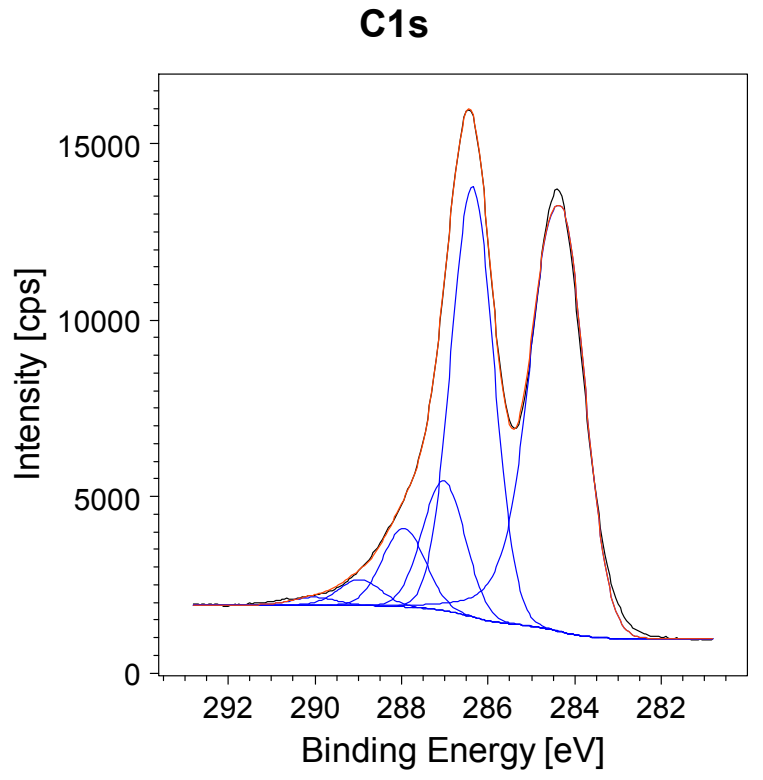

Figure S1. (A) $O 1 s$ and (C) $C 1 s$ spectra of the pristine $G O$ and of graphene reduced with the increasing HI concentrations. An example of deconvolution of $O 1$ s peak is shown in (B) while deconvolution of $C 1 s$ is represented in $(D)$. 
Presence of a graphitic phase suggests the use of a Shirley background for the carbon corelines while linear background was used for the oxygen core-lines. Both the carbon and oxygen spectra were fitted using Gaussian components. As it can be seen at increasing the molar concentration of the HI acid the intensity of the oxidized components of carbon in the range $286-289.5 \mathrm{eV}$ decrease. In agreement with this, a decrease of the oxygen concentration is also observed. 